\title{
The Effect of the Ion Beam Energy on M-plane InGaN Layer Preparation for STEM
}

Trang Nguyen ${ }^{1}$, Brandon Dzuba ${ }^{1}$, Yang $\mathrm{Cao}^{1}$, Alexander Senichev ${ }^{2}, \mathrm{Rosa} \mathrm{Diaz}^{2}$, Geoffrey Gardner ${ }^{2}$, Michael Manfra ${ }^{1,2,3,4}$ Oana Malis ${ }^{1}$

1. Department of Physics and Astronomy, Purdue University, West Lafayette, IN, USA.

2. Birck Nanotechnology Center, West Lafayette, IN, USA.

3. School of Electrical and Computer Engineering, Purdue University, West Lafayette, IN, USA.

4. School of Materials Engineering, Purdue University, West Lafayette, IN, USA.

Nonpolar $\mathrm{AlGaN} / \mathrm{InGaN}$ superlattices are investigated as a strain balanced potential alternative for nonpolar high $\mathrm{Al}$ composition $\mathrm{AlGaN} / \mathrm{GaN}$ for near infrared intersubband absorption [5]. Inhomogeneity and defects appearing during InGaN growth are still challenges to the realization of high performance nitride infrared optoelectronic devices. These defects can be imaged with various TEM imaging modes [1-4]. Nonetheless, damage caused by the sample preparation process reduces the quality of images and raises difficulties to the study of defects in InGaN thin films. We discuss in detail our attempts to prepare cross section TEM samples of $30 \mathrm{~nm}$ m-plane $\operatorname{In}_{\mathrm{x}} \mathrm{Ga}_{1-\mathrm{x}} \mathrm{N}(\mathrm{x}$ $=0.1-0.15)$ using the traditional polishing method followed by ion polishing with various beam energies and the resultant quality of the specimens.

Our InGaN thin films are grown on m-plane free standing GaN substrates by plasma assisted molecular beam epitaxy. These samples are prepared for TEM using traditional methods followed by an ion beam milling process with a Gatan Precision Ion Polishing System II (PIPSII). Prior to the mechanical polishing process, samples are glued together and cured at $160^{\circ} \mathrm{C}$ for 1.5 hours with the M-bond 610 . Then, specimens are pre-thinned using an Allied Optiprep Polishing System with diamond lapping paper over a wide range of roughnesses (0.1-15um), before being attached onto a TEM grid. Subsequently, additional material is removed using a Gatan Dimple Grinder II with 0-4um diamond pastes and $10 \mathrm{gr}$ of weight. At the end of the dimpling process, each sample is around $10 \mathrm{um}$ thick. A series of PIPSII beam energies $(1-5 \mathrm{keV})$ is used on different specimens to create an electron-thin region for TEM imaging. Table 1 summarized these different PIPSII procedures.

Finally, the samples are imaged with an FEI Talos 200kV Microscope with a Super X-EDS detector. Figure 1 shows the STEM images for samples A, B, and C. Figure 2 includes EDS elemental 1D maps for In and Ga of sample B. The bright lines from the top to the bottom of images in Fig. 1 correspond to damage caused by PIPSII process. While sample A shows not only damage but also contamination from the sample preparation process, sample B only shows damages from the ion beam of PIPSII. Moreover, EDS line scans show that the nanostructures in samples B are not related to contamination. However, the origin on these unique structures is still not well understood. Sample C with milder ion polishing procedure shows cleaner images with neither damage nor contamination. The red circles in Figure 1c are electron beam damage from TEM during alignment process. Figure $1 \mathrm{~d}$ is a zoom-in section of $1 \mathrm{c}$. It confirms that the procedure used to make sample $\mathrm{C}$ is optimal for cross section InGaN. No evidence for Indium segregation was found in this sample.

Despite the useful information from TEM including the growth dynamics and structural properties of the material, sample preparation is as crucial as TEM imaging. A good sample preparation process produces a precise and certain TEM characterization of the material. Due to the sensitivity of In atoms in the InGaN thin film, a complex sample preparation process is needed to provide suitable samples for TEM. Using the traditional polishing process, we introduce a systematic way of providing the optimal ion polishing procedure. Our STEM images confirm the reduction of the ion beam damage through a series of samples, and insure we have an optimal recipe for cross section InGaN sample preparation [6]. 
References:

[1] J.J. Jiménez et al., Journal of Alloys and Compounds, 783 (2019), p. 697.

[2] K. Prabakaran et al., Applied Surface Science, 476 (2019), p. 993.

[3] Y. Nanishi et al., Phys. Stat. Sol. (a), 200 (2003), p. 202.

[4] X. Wang. (2018), Characterization of InGaN thin Films and Nanowires by Analytical Transmission Electron Microscopy (Doctoral dissertation). Retrieved from http://etheses.whiterose.ac.uk/21255/.

[5] T. Nguyen et al., Phys. Stat. Sol. (a), 215 (2018), p. 1700828.

[6] T. Nguyen, Y. Cao, B. Dzuba, A. Senichev, O. Malis, and M. Manfra acknowledge funding from the National Science Foundation. TN, YC, and OM acknowledge partial support from NSF grant ECCS-1253720, and DMR1610893. AS and BD were supported from NSF award ECCS-1607173.

\begin{tabular}{|c|c|c|c|}
\hline & A ( $5^{\circ}$ angle $)$ & B (5 $5^{\circ}$ angle $)$ & $\mathrm{C}\left(4^{\circ}\right.$ angle $)$ \\
\hline Eye opens & $5 \mathrm{keV}$ & $3 \mathrm{keV}$ & $3 \mathrm{keV}$ \\
\hline Fringes & $4 \mathrm{keV}$ & $3 \mathrm{keV}$ & $2 \mathrm{keV}$ \\
\hline Hole opens & $3 \mathrm{keV}$ & $3 \mathrm{keV}$ & $1 \mathrm{keV}$ \\
\hline Final polish & $1 \mathrm{keV}, 0.5 \mathrm{keV}, 0.25 \mathrm{keV}$ & $1 \mathrm{keV}, 0.5 \mathrm{keV}, 0.25 \mathrm{keV}$ & $0.5 \mathrm{keV}, 0.25 \mathrm{keV}$ \\
\hline
\end{tabular}

Table 1. Ion beam milling process using a Gatan PIPSII with different beam energy between samples A, $\mathrm{B}$, and $\mathrm{C}$.

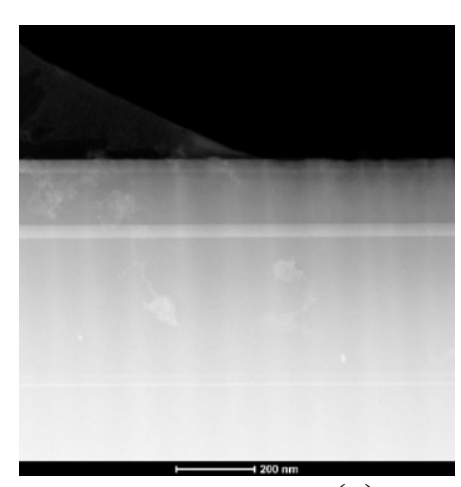

(a)

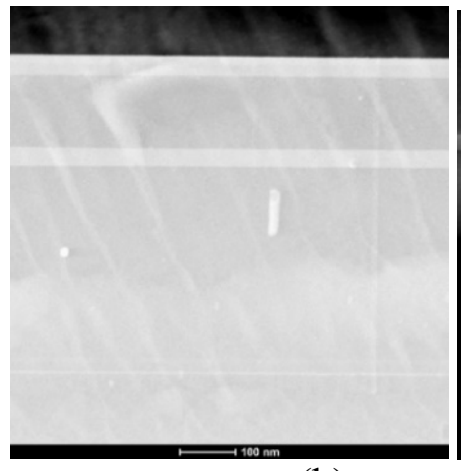

(b)

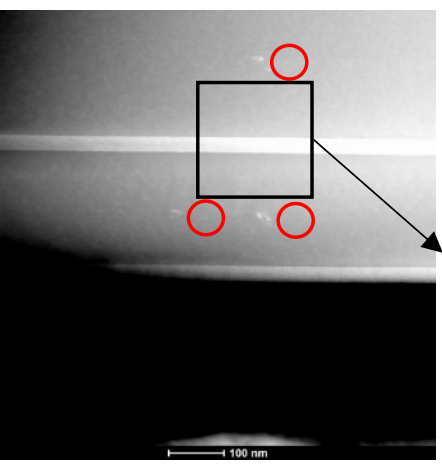

(c)

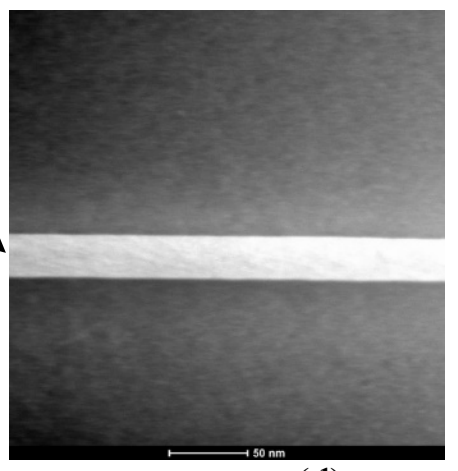

(d)

Figure 1. STEM images of sample A, (a), sample B, (b), and sample C, (c) and (d). The bright vertical/tilted lines are due to damage from the PIPSII process

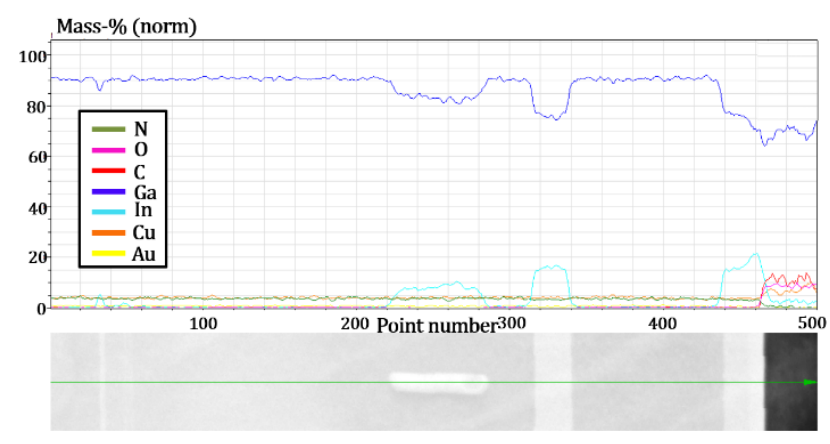

(a)

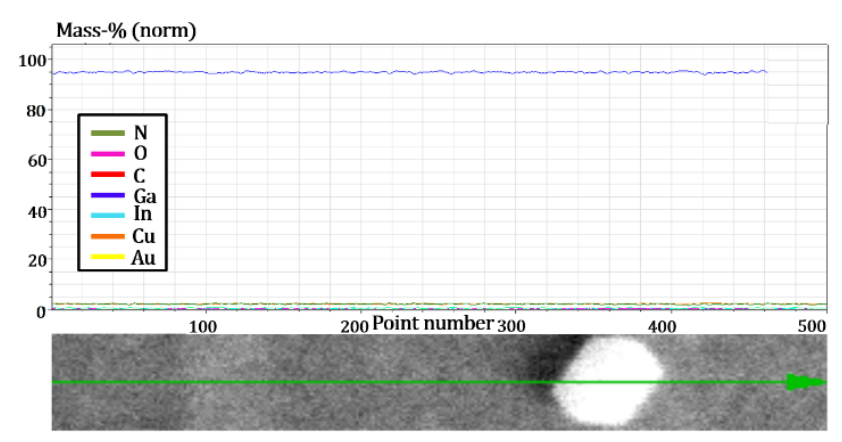

(b)

Figure 2. EDS elemental line scans for unique nanostructures in the GaN layer of sample B. 